\title{
Étude théorique de la reflexion de la houle sur certains obstacles
}

\section{Theoretical study of the reflection of waves from different types of obstacle}

\author{
PAR F. BIESEL et B. LE MEHAUTE;

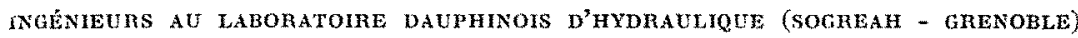

\begin{abstract}
Etude theorique du comportement des ondes de grabité périodiques à deux dimensions en présence d'obstacles, ce mot étant pris dans son sens le plus général et pouvant désigner whe obstruction, un approfondissement, un élargissement...

L'approximation se limite aux phénomènes linéaires. Sans faire appel aux équations intrinsèques da moubement, les calculs sont effectués a laide de nombres complexes, qui permettent de définir la houle par son amplitude (module) et sa phase par rapport à un plan de référence (argument). Les perturbations localisées an voisinage de l'obstacle ne sont pas analysées. Etude des obstacles de formes dissymétriques. Etablissement de la symétrie des effets hydrauliques a partir de lhypothèse de la conservation de l'énergie.

Relations entre les coefficients de transmission et de réflexion (définis par leur amplitude et leur phase par rapport $\dot{a}$ la houle incidente) pour differents tupes d'obstacles conservatifs ou dissipatifs à partir d'hypothèses simples sur la continuité.

Combinaison d'obstactes conservatifs, eotirbes d'agitation et résonance. Combinaison d'obstacles dissipatifs, courbes d'agitation ef «pseudo-résonance».

Annexe : démonstration de M. Meyer sur la symétrie d'effets d'obstacles de formes dissymétriques.
\end{abstract}

Theoretical stady of the two dimensional behaviour of periodic gravity waves in the presence of obstacles. The word obstacles is used in its most general sense and can designate an obstruction, a deepening, a widening. etc.

The approximation is limited to lineat phenomena. Without calling on the equations of motion the calcalations are made with the help of complex numbers, which allow the wave to be defined by its amplitude (module) and its phase in relation to a reference plane (argument). The local perturbations in the neighbonthood of the obstacle are not analysed.

Investigation of asymmetric obstacles. Establishing of symmetry of hydraulic effect, from the hypothesis of conservation of energy.

Relations between the coefficients of transmission and reflection ldefined by their amplitude and phase referred to the incident wave) for different types of conservative or dissipative obstacles, starting from simple hypotheses on continnity.

Combination of conservative obstacles, curbes of agitation and resonance. Combination of dissipatine obstacles, curves of agitation and "pseudo-resonance".

Annex : demonstration by M. Meyer of the symmetry of effect of asymmetrical abstacles. is i

\section{A) DÉFINITION DU PROBLÈME ÉTUDIÉ}

Une partie de cetle élude a fait l'objet d'une communication à la Société Hydrotechnique de France [1]. Elle se limite aux houles périodiques irrotationnelles à deux dimensions en profondeur constante, houles « en canal 》. Nous supposons (en première étape) que le fluide étudié est parfait et que les termes du second ordre par rapport à la cambrure de la houle sont négligeables. Nous restons donc dans le domaine des théories d'u premier ordre, ou «linéaires».

Les obstacles étudiés sont tels qu'ils laissent passer une partie de la houle et en réfléchissent une autre partie. Nous admettrons que ceci se passe sans absorption d'énergie, sauf lorsque nous le précisons, et sans déformation des houles, sauf au voisinage immédiat des obstacles étudies.
L'élude complète des efrets d'un obstacle sur la houle est accessible dans quelques cas simples. Par exemple, les calculs de M. Unsell [47 permettent d'évaluer lès coefficients de transmission et de réflexion d'un obstacle en eau de profondeur infinie, constitué par une paroi verticale conforme à l'un des schémas de la figure 1.

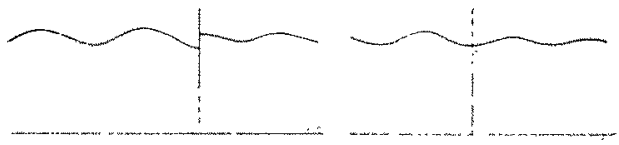

F1G. 1

Les calculs destinés à établir l'équation exacte des mouvements de l'eau dans ce cas sont très complexes et nous ne les étudierons pas ici dans 
le détail. Nous n'en conserverons que les résullats caractéristiques suivants :

$1^{\circ}$ Les ondes incidentes, transmises et refléchies, subissent des déformations notables au voisinage inmédiat de l'obstacle, mais ces deformations disparaissent très rapidement (exponentiellement) dès que l'on s'ćloigne de celui-ci;

$2^{\circ}$ Si l'on considere les ondes incidentes, transmises ef réfléchies, à une distance suffisante de l'obstacle pour qu'elles aient repris le caractire d'ondes périodiques en profondeur constante, la theorie montre que le rapport de l'amplitude de l'onde transmise à celle de l'onde incidente est un certain nombre $\alpha$ indépendant de la valeur absolue des amplitudes et que, de même, le rapport entre l'amplitude de l'onde réflćchie et celle de l'onde incidente est un certain nombre $\beta$. Ceci est d'ailleurs lié au fait que seules les théories linéaires sont utilisées.

D'autre part, les ondes transmises ont un certain décalage de phase, que nous désignerons par $\hat{x}$, par rapport atux ondes incidentes, et de même les ondes refléchies onl un certain décalage $\hat{\beta}$ par rapport aux ondes qui seraient réfléchies par une paroi verticale complete placée au même endroit que la barrière constituant l'obstacle étudié.

Nous admeltrons que ces résultats généraux restent valables pour des obstacles plus profilés que ceux de la figure 1 et en profondeur finie.

De façon à pouvoir étudier non seulement les amplitudes des houles transmises ou réfléchies, mais également leurs phases, nous introduirons un sens et un plan de référence lié à l'obstacle et nous conviendrons de désigner la phase de la houle par celle des amplitudes verticales au droit de ce plan de référence ( ${ }^{\star}$ ). Cet artifice nous permet en particulier de comparer les phases des houles incidentes et réllechies.

Pour wne barrière plane thérique, il ast loul naturel de choisir le plan même de l'obstacle comme plan de référence. On voil alors que si l'obstacle disparait, la houle se propage sans aucune modification, ni d'amplitude, ni de phase; par ailleurs, si l'obstacle tend à obstruer tout le canal, la houle se réfléchit également sans modification d'amplitude ni de phase.

Pour des raisons de simplicité ćvidente, lorsque l'obstacle admettra un plan de symétrie ver- tical, c'est ce plan que nous choisirons comme plan de référence. Nous verrons plus loin que, mème dans le cas des obstacles dissymétriques, il y a intérêt à choisir pour plan de référence un certain plan particulier.

Si nous adoptons pour les houles dudiées une représentation vectoriclle dans le plan des imaginaires (fig. 2), analogue à celle employée pour

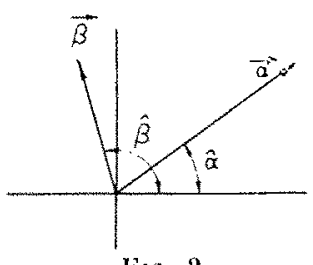

l'étude des courants alternatif's, nous pouvons représenter les coefficients de transmission $\alpha$ el de réflexion $\beta$ par des vecteurs, cest-ádire des nombres complexes $\bar{\alpha}$ et $\bar{\beta}$, représentant à la fois les coefficients de transmission et de réflexion $\alpha$ et $\beta$ par leurs modules et les changements de phase $\hat{\alpha}$ et $\hat{\beta}$ par leurs arguments. Si l'on représente de même par un nombre complexe A la phase et l'amplitude de la houle incidente, les houles transmises et reflechies seront respectivement représentées par les nombres $\bar{x} \mathrm{~A}$ et $\bar{\beta} \mathrm{A}$.

Si l'obstacle est symétrique, il est clair que ces coefficients seront les mèmes, quel que soit le côté d'où vienne la houle incidente. Au contraire, dans le cas d'un obstacle disssymétrique, nous ne pouvons plus conclure à priori. On pourrait par exemple concevoir qu'un obstacle

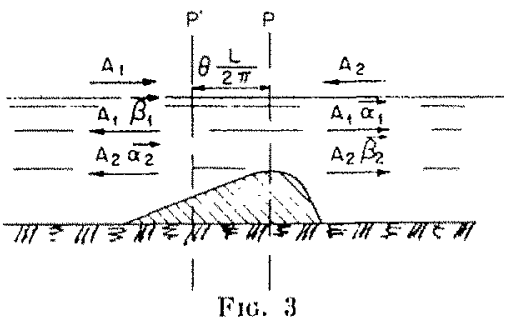

Lel que celui de la figure 3 soit plus rélléchissant dans un sens que dans lautre.

Existe- - il effectivement des obstacles présenlant une telle dissymétrie d'elTets?

C'est cette premiore question de prineipe que nous allons étudier maintenant.

\section{B) ETUDES D'OBSTACLES DYSSYMETRIQUES}

Particularisons par l'indice 1 les coefficients

(*) D'une façon plus précise, il s'agit de la phase des amplitudes verticales d'une houle périodique qui se raccorderait, suffisamment loin de lobstacle, à la houle considérée. On fait en quelque sorte abstraction des perturbations locales dues a l'obstacle pour juger de la phase des honles observées loin de celui-ci. de réflexion el de transmission relatifs aux ondes incidentes venant de gauche et par l'indice 2 ceux relatifs aux ondes incidentes venant de droite (fig. 3 ).

Une houle d'amplitude (imaginatre) $A_{1}$ venant de la gauche donnera done lieu à une transmission d'amplitude $\bar{\alpha}_{1} \mathbf{A}_{1}$ continuant vers la droite 
et $\dot{a}$ une réflexion d'amplitude $\bar{\beta}_{1} A_{1}$ revenant vers la gauche.

La conservation de l'énergie s'exprimera par:

$$
\left|\bar{\alpha}_{1}\right|^{2}+\left|\bar{\beta}_{1}\right|^{2}=\alpha_{1}{ }^{2}+\beta_{1}{ }^{2}=1
$$

On aura de mềme pour l'indice 2 :

$$
\alpha_{2}^{2}+\beta_{2}^{2}=1
$$

Supposons maintenant que des houles de même période abordent simultanément l'ouvrage sur ses deux faces. Soit $A_{1}$ celle venant de gauche et $A_{2}$ celle venant de droite. Ecrivons que l'énergie est conservée :

$$
\begin{aligned}
& \quad\left|A_{1}\right|^{2}+\left|A_{2}\right|^{2}=\left|A_{1} \bar{\beta}_{1}+A_{2} \bar{x}_{2}\right|^{2}+\left|A_{1} \bar{\alpha}_{1}+A_{2} \bar{\beta}_{2}\right|^{2} \\
& =\left|A_{1}\right|^{2}\left(\beta_{1}^{2}+\alpha_{1}^{2}\right)+\left|A_{2}\right|^{2}\left(\alpha_{2}^{2}+\beta_{2}^{2}\right) \\
& -2\left|A_{1}\right|\left|A_{2}\right|\left[\beta_{1} \alpha_{2} \cos \left(\widehat{A}_{1}+\hat{\beta}_{1}-\widehat{A}_{2}-\hat{\alpha}_{2}\right)\right. \\
& \left.\quad+\beta_{2} \alpha_{1} \cos \left(\widehat{A}_{1}+\bar{\alpha}_{1}-\widehat{A}_{2}-\hat{\beta}_{2}\right)\right]
\end{aligned}
$$

On en déduit, compte tenu des relations (1) et (2) :

$$
\begin{aligned}
& \beta_{1} z_{2} \cos \left(\widehat{A}_{1}-\widehat{A}_{2}+\hat{\beta}_{1}-\hat{x}_{2}\right) \\
& +\beta_{2} \alpha_{1} \cos \left(\widehat{A}_{1}-\widehat{A}_{22}+\hat{x}_{1}-\hat{\beta}_{22}\right)=0
\end{aligned}
$$

Supposons tout d'abord $\widehat{A}_{1}-\widehat{A}_{2}=0$, puis $\hat{A}_{1}-\hat{A}_{2}=\pi / 2$.

Dans ces deux cas, la relation (4) doit être vérifiée. Elle devient alors :

$$
\begin{aligned}
& x_{2} \beta_{1} \cos \left(\hat{\alpha}_{2}-\hat{\beta}_{1}\right)+\beta_{2} \alpha_{1} \cos \left(\hat{\beta}_{2}-\hat{x}_{1}\right)=0 \\
& x_{2} \beta_{1} \sin \left(\hat{\alpha}_{2}-\hat{\beta}_{1}\right)+\beta_{2} \alpha_{1} \sin \left(\hat{\beta}_{2}-\hat{\alpha}_{1}\right)=0
\end{aligned}
$$

Soit encore :

$$
\begin{aligned}
& \frac{\alpha_{2}}{\beta_{2}} \cos \left(\hat{\alpha}_{2}-\hat{\beta}_{1}\right)+\frac{\alpha_{1}}{\beta_{1}} \cos \left(\hat{\beta}_{2}--\hat{\alpha}_{1}\right)=0 \\
& \frac{\alpha_{2}}{\beta_{2}} \sin \left(\hat{\alpha}_{2}-\hat{\beta}_{1}\right)+\frac{\alpha_{1}}{\beta_{1}} \sin \left(\hat{\beta}_{2}-\hat{\alpha}_{1}\right)=0
\end{aligned}
$$

On en déduit la condition :

$$
\begin{aligned}
\cos \left(\hat{\alpha}_{2}-\hat{\beta}_{1}\right) & \sin \left(\hat{\beta}_{2}-\hat{\alpha}_{1}\right) \\
& -\cos \left(\hat{\beta}_{2}-\hat{\alpha}_{1}\right) \sin \left(\hat{\alpha}_{2}-\beta_{1}\right)=0
\end{aligned}
$$

Soit encore :

$$
\hat{\beta}_{2}-\hat{\alpha}_{2}+\hat{\beta}_{1}-\hat{\alpha}_{1}=\pi, \quad \text { à } 2 k \pi \text { près }
$$

$\left[\alpha_{1}, \alpha_{2}, \beta_{1}, \beta_{2}\right.$ étant par définition positifs, la solution :

$$
\hat{\alpha}_{2}-\hat{p}_{1}=\hat{\beta}_{2}-\hat{\alpha}_{1}+2 k \pi
$$

est éliminée, cf. équations (6) ].

Supposons qu'au lieu du plan de référence $\mathrm{P}$ on choisisse un plan $P^{\prime}$ situé à $\theta(L / 2 \pi)$ vers la gauche. Les décalages de phase à la transmission sont évidemment inchangés; par contre, les décalages de phase à la réflexion par rapport aux ondes incidentes sont changés de \pm 20 , suivant le sens d'où proviennent les ondes incidentes. $\hat{\beta}_{1}$ et $\hat{\beta}_{2}$ ayant ainsi des variations opposées, on voit que la condition ci-dessus est bien invariante pour un changement de plan de référence. Nous pourrons, en particulier, choisir ce dernier de façon que l'on ait :

$$
\hat{\beta}_{1}-\hat{\alpha}_{1}=\hat{\beta}_{2}-\hat{\alpha}_{2}=\frac{\pi}{2}
$$

à $k \pi$ près.

Une infinité de plans de référence satisfont cette équation et sont distants les uns des autres d'un quart de longueur d'onde. Nous les appellerons plans "normaux », pour l'obstacle et la longueur d'onde de houle considérés.

Dans le cas d'un obstacle symétrique, un de ces plans sera toujours confond $u$ avec le plan de symétrie : c'est celui que nous adopterons définitivement, ainsi que nous l'avons dit plus haut. Dans le cas d'un obstacle dissymétrique, nous choisirons si possible, comme plan de référence, le plan normal le plus central possible par rapport à l'obstacle. Dans le cas des obstacles étendus, cette définition pourra évidemment donner lieu à des ambiguïtés d'ailleurs sans conséquences pratiques.

En portant la relation (8) dans (6), il vient:

$$
\frac{\alpha_{2}}{\beta_{2}}=\frac{\alpha_{1}}{\beta_{1}}
$$

On en déduit immédiatement, compte tenu des relations (1) et (2) :

$$
\alpha_{1}=\alpha_{2} \quad \beta_{1}=\beta_{2}
$$

En conclusion, on peut énoncer le théorème suivant :

"Tout obstacle, même dissymétrique, a des coefficients de transmission et de réflexion dont la valeur absolue ne dépend pas du sens de la houle incidente.

Ce résultat a été trouvé par ailleurs d'une manière tout à fait différente en faisant intervenir 
les équations intrinsèques du phénomène par KrEISEL [3].

M. Meyer a aussi démontré d'une façon plus générale que :

$$
\begin{aligned}
& \bar{\alpha}_{1}=\bar{\alpha}_{2} \\
& \bar{\beta}_{1}=\bar{\beta}_{2}
\end{aligned}
$$

II n'existe donc rigoureusement aucune dis- symétrie d'effet au premier ordre d'approximation.

La condition (8) devient donc dans tous les cas :

$$
\hat{\alpha}-\hat{\beta}=(\pi / 2) \text { à } k \pi \text { pris. }
$$

La démonstration de M. Mirys est donnée en annexe.

\section{C) HYPOTHESES COMPLEMENTAIRES}

Si l'on considère un obstacle du type barrière verticale, on remarque que lorsque l'obstruction du canal est totale, il y a réflexion totale sans changement de phase; autrement dit on a :

$$
\begin{aligned}
|\beta|=1, \quad \hat{\beta}= & 0, \quad \text { soit } \bar{\beta}=1 \\
& (\text { pour mémoire }: \bar{\alpha}=0)
\end{aligned}
$$

Lorsque, au contraire, la barrière s'efface complètement, il y a transmission totale sans changement de phase; on a done :

$$
\begin{aligned}
&|x|=1, \quad \bar{x}=0, \quad \text { soit } \bar{x}=1 \\
&(\text { pour mémoire }: \bar{\beta}=0)
\end{aligned}
$$

Ces remarques suggèrent que, pour ce type d'obstacle, il y a une relation imposée entre les déphasages et les valeurs absolues des coefficients de réflexion et de transmission. Ainsi que nous allons le montrer, des considérations physiques simples, qui, bien qu'hypothétiques, sont néanmoins très plausibles, permettent effeclivement de définir de telles relations.

Ceci nous amène à établir une classification particulière des différents obstacles possibles.

\section{I}

\section{Obstacle du type " obstruction localisée"}

\section{$1^{\circ}$ Obstacles ne Consomman't pas D'Énergie:}

Nous rappelons qu'un obstacle, au sens usuel du terme, sera dit du type \& obstruction " lorsqu'il réalisera une restriction du passage offert aux houles, dans un canal d'étude par exemple. Cette définition semble a priori inutile, mais nous verrons plus loin qu'il y a certains types de discontinuités du canal qui peu- vent se comporter comme des obstacles, partiellement réfléchissants, sans pour cela avoir le caractère d'une obstruction. Au contraire, il peut s'agir d'un élargissement ou d'un approfondissement local.

Par « localisé », nous entendons que les objets constituant l'obstacle ne doivent occuper qu'une longueur très faible par rapport à la longueur d'onde dans le sens de la propagation de la houle.

Le type même de l'obstruction localisée est la barrière verticale plane sans épaisseur.

Notre première hypothèse, liée au fait que l'obstacle est du type obstruction, sera: s'il y a un décalage de phase pour la transmission, ce sera dans le sens d'un retard. En effet, l'obstruction en réduisant les sections de passage provoquera des augmentations de vitesses, donc des augmentations des efrets d'inertie (comparer avec des augmentations de self en électricité). De même une réduction de la profondeur sur un certain parcours (obstacle non localisé) provoque un retard de phase car la célérité est plus faible au-dessus de l'obstacle.

Notre hypothèse sera done:

$$
-\pi<\hat{x}<0 \text { à } 2 k \pi \text { près }
$$

Notre seconde hypothèse, liée an fait que l'obstacle est localisé, sera: les houles asymptotiques (incidente, réfléchic et transmise), supposées prolongées jusqu'à l'obstacle, s'y raccordent en débit.

L'hypothèse de la continuité des débits est naturelle, car si elle n'était pas vérifice dans la région de l'obstacle on accumulerait et restituerait alternativement un certain débit, ce qui semble a priori impossible puisque l'obstacle n'occupe qu'une région pratiquement sans étendue. Il est vrai cependant qu'un obstacle, même très localisé (plaque mince par exemple) peut déformer la houle dans une région voisine qui, en fait, 
aura une étendue de l'ordre de grandeur de quelques profondeurs $\left({ }^{\star}\right)$.

Celte condition nous permet d'ctablir une relation entre $\bar{\alpha}$ et $\bar{\beta}$, a partir de l'expression des fonctions potentielles des mouvements de chaque côté de l'obstacle, caractérisés par les indices 1 et 2 [2] (notations habituelles):

$$
\begin{aligned}
& p_{1}=-a \frac{k}{m} \frac{\operatorname{ch} m(h-y)}{\operatorname{sh} m h} \sin (k t-m x) \\
& -n \beta \frac{k}{m} \frac{\operatorname{ch} m(h-y)}{\operatorname{sh} m h} \sin (k t+m x+\hat{\beta})
\end{aligned}
$$

$\varphi_{2}=-a+\hat{\alpha} \frac{k}{m} \frac{\operatorname{ch} m(h-y)}{\operatorname{sh} m h} \sin (k t-m x+\hat{x})$

avec :

$$
m=\frac{2 \pi}{\mathrm{L}} \quad \text { et } \quad k=\frac{2 \pi}{\mathrm{T}}
$$

En prenant l'obstacle comme origine des absçisses, l'égalité des débits se met successivement sous la forme :

$$
\left[u_{1}(y)\right]_{x=0}=\left[u_{2}(y)\right]_{x=0}
$$

soit :

$$
\left(\frac{\partial \varphi_{1}}{\partial x}\right)_{x=0}=\left(\frac{\partial \varphi_{2}}{\partial x}\right)_{x=0}
$$

En faisant :

$$
k t=\pi / 2 \quad \text { et } \quad k t=0,
$$

on en déduit les relations :

$$
\begin{aligned}
& 1-\beta \cos \hat{\beta}=\alpha \cos \hat{\alpha} \\
& -\beta \sin \hat{\beta}=\alpha \sin \hat{\alpha}
\end{aligned}
$$

d'où finalement :

$$
\bar{\alpha}+\bar{\beta}=1
$$

Les hypothèses (1) et (2) jointes à celle de la conservation de l'énergie :

$$
|\alpha|^{2}+|\beta|^{2}=1
$$

permettent, tout en vérifiant incidemment la loi :

$$
\hat{\beta}-\hat{x}= \pm \pi / 2
$$

(*) On peut en effel montrer que ces déformations sont une somme de termes décroissant exponentiellement très rapidement lorsque l'on s'éloigne de l'obstacle, le taux de décroissance etant lié à la protondeur.

Notre raisonnement sera donc valable à la limite pour les ondes très longues par rapport a la profondeur. II sem au contraire douleux pour les profondeurs relatives plus importantes. Cette note pessimiste est compenséc par le fail que l'analyse exacte du cas de la plaque plane verticale en profondeur infinie montre que l'hypothèse de la continuité se trouve (peut-être fortuitement) satisfaite. trouvée précédemment, de déterminer les quantités $|\alpha|,|\beta|, \bar{\alpha}$ et $\bar{\beta}$, à condition de connaitre seulement l'une d'entre elles.

Cette détermination peut se faire géométriquement par construction d'un triangle rectangle de cotes $\bar{\alpha}$ et $\bar{\beta}$ et d'hypoténuse 1 (voir fig. 4).

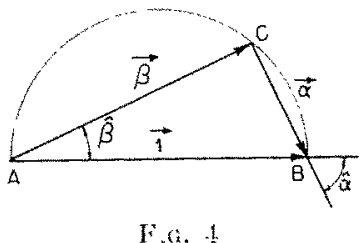

Tous les obstacles de ce type, c'estua-dire satisfaisant aux hypothèses (1) et (2), sont done définis par un seul paramètre, par exemple $\bar{\beta}$, et l'on a:

$$
\begin{aligned}
& \hat{\alpha}=\hat{\beta}-\pi / 2 \\
& |\alpha|=\sin \hat{\beta} \\
& |\beta|=\cos \hat{\beta}
\end{aligned}
$$

\section{2" OBSTACLES DU TYPF OBSTRUCTIONS LOCALES ARSORBANT L'ÉNERGITE.}

Les obstacles étudiés dans ce paragraphe étant également du type obstruction locale, nous conserverons les hypothèses (1) et (2) en y adjoignant la condition de non-conservation de l'énergie :

$$
|\alpha|^{2}+|\beta|^{2}<1
$$

Si l'on prend une représentation graphique analogue à celle de la figure 4 , on voit que le triangle $A B C$ a son sommet $C$ à l'intérieur du cercle de diamètre $\mathrm{AB}$ (fig. 5 ).

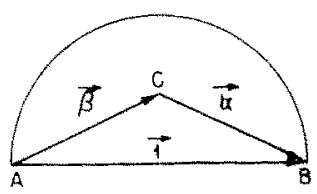

Fia. 5

Pour une valeur donnée de $\alpha$, de $\beta$ ou de $\alpha / \beta$, on voit qu'il $y$ a un maximum d'absorption quand $C$ vient sur le diamètre $A B$.

$\mathrm{Si}$ la transmission est nulle, la réflexion se fait sans perte d'énergie et, mutatis mutandis, si la réflexion est nulle, la transmission se fait sans perte d'énergie.

On voit dono que, sous réserve du bien-fondé de nos hypothèses:

- Une obstruction localisée ne peut absorber plus d'un certain quota d'énergie et ce quota 
limite est lui-même fonction du rapport des coefficients de réflexion et de transmission. En particulier :

a) Aucune énergie ne peut être absorbée si l'un de ces coefficients $\alpha$ et $\beta$ est nul (l'autre étant par conséquent égal à l'unité);

b) La proportion maximum maximorum ne peut être atteinte que si les coefficients $\alpha$ et $\beta$ sont égaux. Leur valeur commune est alors $1 / 2$. Cette proportion est alor's de $50 \%$.

Notons incidemment que ceci confirme et précise le fait connu qu'il n'est pas possible d'absorber complètement une houle sur une longueur trop restreinte avec des moyens purement passifs. Par exemple la règle pratique de notre Iaboratoire est qu'au moins une longueur d'onde est nécessaire pour faire un amortisseur acceptable.

Lorsque le point $C$ se trouvera sur $A B$, nous dirons que l'obstacle est purement résistant (par analogie avec l'électricité où une résistance pure provoque des pertes d'énergie et pas de déphasage). Le type d'obstruction localisée purement résistante est la plaque verticale en matériau perméable obturant tout le canal.

L'essentiel est d'ailleurs que les efforts s'opposant au passage de leau soient proportionnels aux vitesses et que les effets d'inertie soient négligeables dans l'obstacle.

\section{II}

\section{Obstacle du type \\ " approfondissement localisé "}

Il est évident que pour aroir une efficacité réelle, un approfondissement ne devra pas être trop itroit; néanmoins pour qu'il justifie l'épithète localisé, nous admẹttons qu'il ne doit occuper qu'une longueur petite par rapport à celle des ondes.

Dans le cas de ce type d'obstacle, l'hypothèse (1) est changée et devient :

$$
0<\approx<\pi \quad \text { à } 2 k \pi \text { près }
$$

L'hypothèse (2) reste inchangée.

Lal figure 4 est remplacée par la figure 6. No-

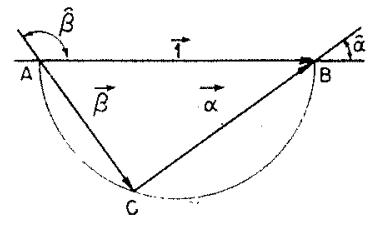

FIG. 6 tons d'ailleurs que, sauf pour les ondes très longues en faible profondeur, ce type d'obstacle ne peut donner des réflexions très fortes.

\section{III}

\section{Obstacle du type "élargissement brusque"}

Si un canal étroit est mis en communication en une de ses sections avec un vaste plan d'eau (fig. 7), le niveau en ce point tendra à rester constant et une réflexion avec changement do signe se produira.

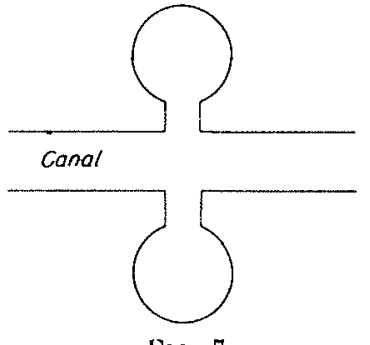

Fig. 7

Pour ce type d'obstacle, la condition de conlinuité des débits ne s'applique évidemment pas puisque les plans d'eau sont au contraire de vastes réservoirs d'accumulation. Au contraire, pour le type parfait de ce genre d'obstacle (dont il est difficile de voir une réalisation mécanique simple), le niveau serait maintenu constant et les coefficients $\approx$ et $\beta$ vaudraient :

$$
y=0 \quad \beta=-1
$$

En réalité, un obstacle constituć, comme le montre la ligure 7 , ne sera jamais parfait car if $\mathrm{y}$ aura toujours une fluctuation résiduelle de niveau à l'entrée des bassins el de plus une certaine déperdition d'énergie par rayonnement dans ces derniers.

En résumé, il ne semble pas possible de faire des hypothèses sérieuses sur les phases des ondes transmises et rélléchies. Cependant, le cas de l'élargissement brusque est important en pratique; c'esl pourquoi il peut être intéressant de poser un schéma nécessairement un peu arbiIraire que l'on pourra utiliser avec précaution dans les études ultérieures. Nous proposerons les hypothèses suivantes :

1" La transmission se fait toujours sans décalage de phase el la réflexion toujours avec changement de signe;

$2^{\prime \prime}$

$$
|\alpha|+|\beta|=1
$$


On en tire le schéma de la figure 8 . Ce schéma

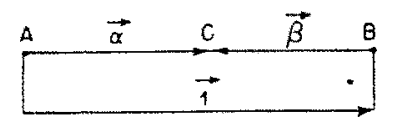

Fic, 8 rend compte d'une perte d'énergie, sauf pour la transmission parfaite $(\beta=-1)$ et la réflexion parfaite $(\alpha=1)$; on sait d'ailleurs que ce dernier cas ne peut être réalisé que très approximativement.

\section{D) COMBINAISONS D'OBSTACLES}

Après avoir établi les équations relatives aux obstacles isolés, nous allons maintenant étudier l'effet combiné d'obstacles des types considérés disposés les uns derrière les autres. Nous supposerons pour nos calculs que ces obstacles sont toujours suffisamment éloignés les uns des autres pour qu'il n'y ait pratiquement pas d'interaction des zones de perturbation entourant chacun d'eux. Cette condition est théoriquement toujours réalisable à la précision voulue, car les positions relatives ne sont en général définies qu'à des multiples de la demi-longueur d'onde près.

\section{Agitation entre deux obstacles quelconques}

Les coefficients de transmission et de réflexion sont relatifs à chaque obstacle (et à son plan de symétrie) considéré isolément. Les grandeurs $\mathrm{A}_{n}, \mathrm{~B}_{n}, \mathrm{C}_{n}$ et $\mathrm{D}_{n}$ représentent les houles et leurs phases relatives à l'obstacle $n$; de même $A_{i k-1}$, $\mathrm{B}_{n-1}, \mathrm{C}_{n-1}$ et $\mathrm{D}_{n-1}$ représentent les houles et leurs phases relatives à l'obstacle $n-1$ (fig. 9).

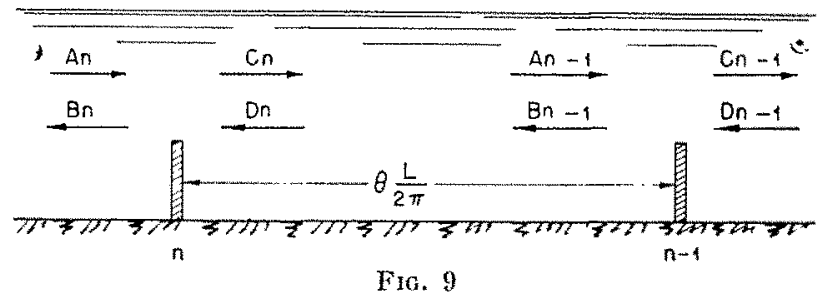

On a les relations suivantes :

$$
\begin{aligned}
& \mathrm{B}_{n}=\bar{\beta}_{n} \mathrm{~A}_{n}+\bar{\alpha}_{n} \mathrm{D}_{n} \\
& \mathrm{C}_{n}=\bar{\alpha}_{n k} \mathrm{~A}_{n}+\bar{\beta}_{n} \mathrm{D}_{n k} \\
& \mathrm{~B}_{n-1}=\bar{\alpha}_{n-1} \mathrm{D}_{n-1}+\bar{\beta}_{n-1} \mathrm{~A}_{n-1} \\
& \mathrm{C}_{n-1}=\bar{\alpha}_{n-1} \mathrm{~A}_{n-1}+\bar{\beta}_{n-1} \mathrm{D}_{n-1} \\
& \mathrm{C}_{n}=\bar{r}_{n, n-1} \mathrm{~A}_{n-1} \\
& \mathrm{~B}_{n-1}=\bar{r}_{n, n-1} \mathrm{D}_{n}
\end{aligned}
$$

avec :

$$
\bar{r}_{n, n-1}=p e^{i \theta}
$$

et :

$$
\theta=2 \pi \frac{d}{\mathrm{~L}}
$$

$d$ étant la distance séparant les deux obstacles.

Ce qui donne, en fonction de $A_{n}$ et $D_{n-1}$ :

$$
\begin{aligned}
& \mathrm{D}_{n}=\frac{\mathrm{B}_{n-1}}{\bar{r}_{n, n-1}}=\frac{\bar{\alpha}_{n} \bar{\beta}_{n-1} A_{n}+\bar{r}_{n, n-1} \bar{\alpha}_{n-1} \mathrm{D}_{n-1}}{\bar{\Gamma}_{n, n-1}{ }^{2}-\bar{\beta}_{n} \bar{\beta}_{n-1}} \\
& A_{n-1}=\frac{C_{n}}{\bar{r}_{n, n-1}}=\frac{\bar{\alpha}_{n-1} \bar{\beta}_{n} \mathrm{D}_{n-1}+\bar{\alpha}_{n} \bar{r}_{n, n-1} \mathrm{~A}_{n}}{\bar{\Gamma}_{n, n-1}{ }^{2}-\bar{\beta}_{n} \bar{\beta}_{n-1}} \\
& \left(\bar{r}_{n, n-1}{ }^{2} \bar{\beta}_{n-1}-\bar{\beta}_{n-1}{ }^{2} \bar{\beta}_{n}+\bar{\alpha}_{n-1}{ }^{2} \bar{\beta}_{n}\right) \mathrm{D}_{i n-1} \\
& C_{n-1}=\frac{+\bar{r}_{n+n-1} \bar{\alpha}_{n 2} \bar{\alpha}_{n-1} A_{n}}{\bar{r}_{n, n-1}{ }^{2}-\bar{\beta}_{n} \bar{\beta}_{n-1}} \\
& \left(\bar{r}_{n, n-1}{ }^{2} \bar{\beta}_{n}-\bar{\beta}_{n}^{2} \beta_{n-1}+\bar{\alpha}_{n}^{2} \ddot{\beta}_{n-1}\right) A_{n} \\
& \mathrm{~B}_{n}=\frac{+\bar{r}_{n, n-1} \bar{\alpha}_{n-1} \mathrm{D}_{n-1}}{\bar{r}_{n, n-1}{ }^{2}-\bar{\beta}_{n} \bar{\beta}_{n-1}}
\end{aligned}
$$

On peut de suite voir que l'agitation entre les obstacles $n$ et $n-1$ peut être calculée quand on connait $A_{n}$ et $D_{n-1}$ et qu'elle est généralement infinie pour :

$$
\bar{\Gamma}_{n, n-1}{ }^{2}-\bar{\beta}_{n} \bar{\beta}_{n-1}=0
$$

En pratique, le frottement interviendrait pour limiter l'amplitude de l'agitation.

\section{II}

\section{Agitation entre deux obstacles identiques soumis à une seule houle incidente}

Les formules précédentes deviennent avec $\mathrm{D}_{n-1}=0$, et en posant :

$$
\bar{\alpha}_{n-1}=\bar{\alpha}_{n}=\bar{\alpha}
$$




$$
\begin{aligned}
& \ddot{\beta}_{n-1}=\bar{\beta}_{n}=\bar{\beta} \\
& \mathrm{D}_{1}=\frac{\bar{\alpha} \bar{\beta} \mathrm{A}_{1}}{\bar{r}^{2}-\bar{\beta}^{2}} \\
& \mathrm{C}_{1}=\frac{\bar{\alpha} \bar{r}^{2} \mathrm{~A}_{1}}{\bar{r}^{2}-\bar{\beta}^{2}}
\end{aligned}
$$

An centre $O$, l'agitation devient :

$$
\mathrm{A}_{y}=\mathrm{C}_{0}+\mathrm{D}_{0}=\bar{r}^{1 / 2} \mathrm{D}_{1}+\bar{r}^{-1 / 2} \mathrm{C}_{1}
$$

Soit, après simplification :

$$
A_{g}=\frac{\bar{r}^{1 / 2} \bar{\alpha} A_{1}}{\bar{r}-\bar{\beta}}
$$

\section{$1^{\circ}$ Obstacles ne consommant Pas d'Énergre :}

Compte tenu des hypothèses sur les obstacles localisés ne consommant pas d'énergie, à savoir :

$$
\begin{aligned}
& \vec{z}=\sin \hat{\beta} \\
& \bar{\beta}=\cos \hat{\beta}
\end{aligned}
$$

Cette expression complexe s'écrit en passant aux arguments :

$$
A_{y}=\frac{\sin \hat{\beta} A_{1}}{\sqrt{1+\cos ^{2} \hat{\beta}-2 \cos (0-\hat{\beta}) \cos \hat{\beta}}}
$$

avec :

$$
\widehat{\theta}=2 \pi(d / L)
$$

$d$ étant la distance entre les deux obstacles.

L'agitation sera done maximum pour les valeurs de $\widehat{\theta}$ :

$$
\hat{0}=\hat{\beta}+2 k \pi
$$

L'agitation est alors :

$$
A_{1}=\frac{\bar{\alpha} A_{1}}{1-\bar{\beta}}
$$

ce qui se traduit par l'expression :

$$
A_{M}=\frac{\sin \hat{\beta} \cdot A_{1}}{1-\cos \hat{\beta}}
$$

Cette relation donne la valeur de lagitation de résonance en fonction de $\widehat{\beta}$.

Dans ce cas, la réflexion sur l'ensemble des deux obstacles est nulle.

Il est à remarquer que quand $\hat{\mathrm{\beta}}$ tend vers 0 (ce qui correspond physiquement d'après les hy-

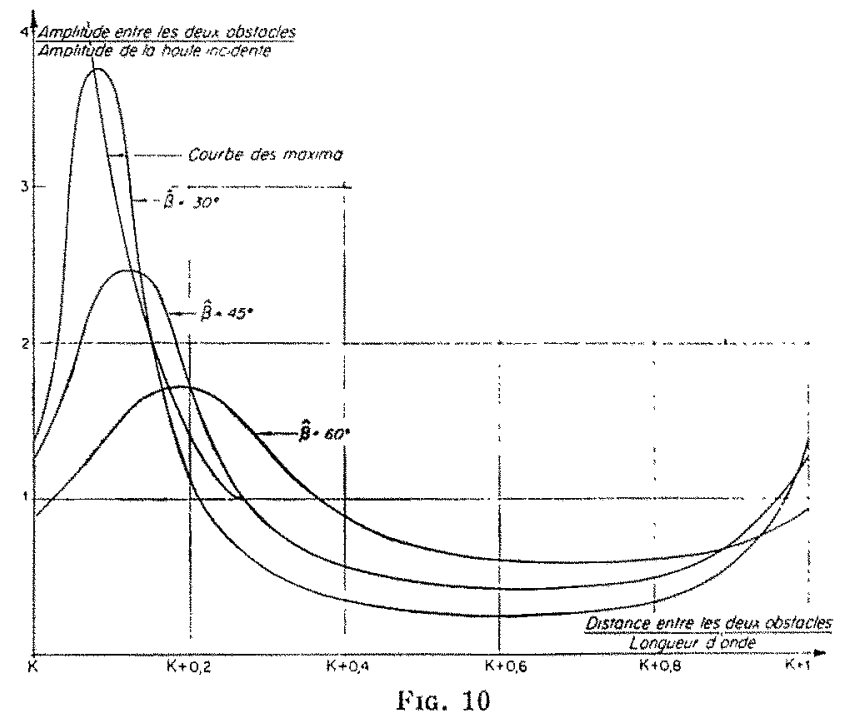

pothèses antérieures à la fermeture complète), l'agitation de résonance tend vers l'infini.

Les courbes théoriques (10) données par la formule (4) mettent en évidence l'accroissement de la sélectivité de résonance quand $\tilde{\beta}$ diminue. Par contre, l'agitation de résonance croit simultanément.

Ceci se traduit physiquement par le fait que plus les obstacles limitent le passage aux houles, moins les eaux comprises entre eux risquent de résonner sous l'action de la houle du large, mais plus l'agitation peut être très grande en cas de résonance. En fait, elle sera cependant limitée par les pertes d'énergie dues aux frottements se produisant d'une part dans la darse, et à la transmission a travers l'obstacle d'autre part.

L'agitation minimum a lieu pour des valeurs de :

$$
\theta=\beta+(2 k+1) \pi
$$

Elle est alors égale à :

$$
A_{m}=\frac{\bar{\alpha} A_{1}}{1+\bar{\beta}}
$$

L'amplitude s'écrit avec les mêmes hypothèses:

$$
A_{n}=\frac{\sin \hat{\beta} A_{1}}{1+\cos \hat{\beta}}
$$

Relations remarquables. - Il est intéressant de dégager quelques formules remarquables des expressions (6) et (7).

a) Le produit $A_{\mathrm{M}} \times A_{m}$ devient successivement :

$$
A_{\mathrm{X}} \times A_{m}=\frac{\bar{\alpha}^{2}}{1 \cdots \bar{\beta}^{2}} A_{1}^{2}=A_{1}^{2}
$$


avec :

$$
\bar{\alpha}^{2}+\tilde{\beta}^{2}=1
$$

Il convient de préciser que l'égalité :

$$
\mathrm{A}_{\mathrm{M}} \times \mathrm{A}_{m}=\mathrm{A}_{1}{ }^{2}
$$

n'est valable qu'en fluide parfait.

b) $\bar{z}$ et $\bar{\beta}$ sont obtenus à partir des expressions:

$$
\begin{aligned}
& \bar{\alpha}=\sin \hat{\beta}=\frac{A_{\mathrm{M}} \times A_{m}}{2\left(A_{\mathrm{MI}}+A_{m}\right)} \\
& \vec{\beta}=\cos \hat{\beta}=\frac{A_{\mathrm{M}}-A_{m}}{A_{\mathrm{MI}}+A_{m}}
\end{aligned}
$$

Ces formules permettent d'avoir expérimentalement $\bar{\alpha}$ et $\bar{\beta}$ à partir de $A_{M}$ et $A_{m}$, au bien-fondé près des hypothèses antérieures.

c) Il pourra ètre intéressant par la suite de faire l'hypothèse, peut-être un peu simpliste, que les énergies transmises et réfléchies sont respectivement proportionnelles à l'ouverture $o$ et à la fermeture $(l-o)$ ( $l$ étant la largeur du canal), les valeurs de $\bar{\alpha}$ et $\bar{\beta}$ seront en première approximation respectivement égales à :

$$
\begin{aligned}
& \bar{\alpha}=\sqrt{o / l} \\
& \bar{\beta}=\sqrt{(l-o) / l}
\end{aligned}
$$

$2^{\circ}$ Obstacles localisés PUREMENT RÉSISTANTS :

Si nous posons :

$$
\alpha+\beta=1
$$

l'expression de l'agitation devient, compte tenu de la valeur de $\bar{\beta}=0$ :

$$
A_{y}=\frac{\bar{r}^{1 / 2}(1-\bar{\beta}) A_{1}}{\sqrt{1+\beta^{2}-2 \beta \cos 0}}
$$

D'où les courbes théoriques donnant la valeur de l'agitation en fonction de $\alpha$ et $\beta$. Il est alors à remarquer que l'agitation dans la darse est toujours inférieure à l'agitation au large et ne l'égale

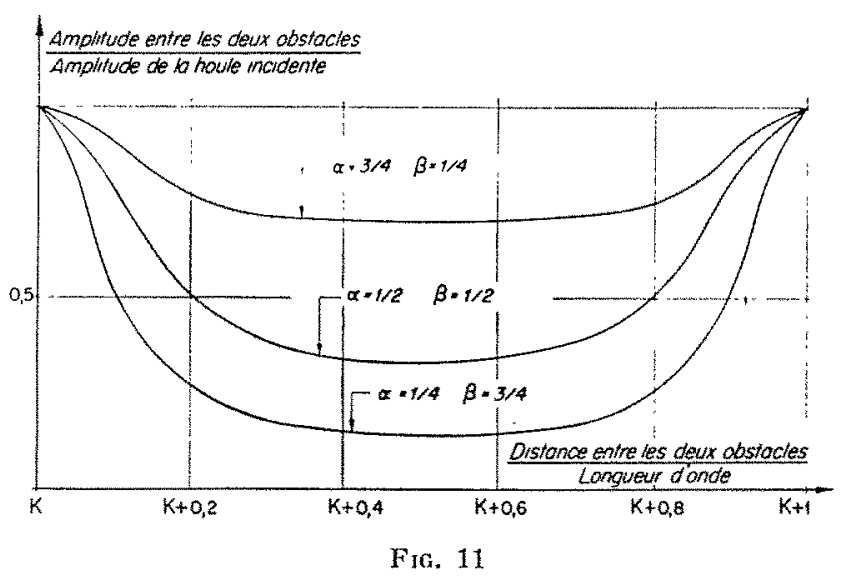

qu'à la résonance (fig. 11) (*). Il faut donc toujours être très prudent avant d'attribuer à une digue perméable la présence de seiches d'une grande amplitude dans un bassin.

(*) Il s'agit plutôt d'une a pseudo-résonance ».

\section{Bibliographe}

1. Breser. - Etude théorique de la réflexion de la houle sur certains obstacles.

La Houille Blanche, $\mathrm{n}^{\circ} \mathrm{A}$, mars 1952.

2. Bresez. - Equations générales de la houle irrégulière. La Honille Blanche, $\mathrm{n}^{\circ} 3$, mai-juin 1952 .
3. KREISEL. - Surface waves. Quaterly of Applied Mathematics, Vol. VII, $\mathrm{n}^{\circ} 1,1949$.

4. URSELL. - The effect of a vertical barrier on waves in deep water. Admiralty research laboratory. TeddingtonMadsx, décembre 1945 . 


\section{ANNEXE}

Symétrie du coefficient (complexe) de transmission des houles à travers un obstacle quelconque

par R. MEYER,

INGŔNEUR AU LABORATOHE DAUPHINOIS D'HYDRAULYUE

Nos notations et hypothèses seront celles de l'article de MM. Biesel et Le MéhautÉ, sauf lorsque nous le préciserons.

Nous allons montrer que pour tous les obstacles (dissipatifs ou non), mais autour desquels l'écoulement satisfait à certaines conditions, on a :

$$
\alpha_{1}=\alpha_{2}
$$

Les nombres $\alpha_{1}, \alpha_{2}$, sont des nombres complexes. Disons de suite que cétte propriété découle directement du théorème de réciprocité. Nous nous permettons de rappeler au passage que la notion de réciprocité est une notion fondamentale et bien connue en physique théorique. Elle intervient en outre en élasticité (théorème de MaxwelL), en électricité dans la théorie des multipoles passifs et d'une façon générale dans la plupart des systèmes linéaires où il n'y a pas de convexion du milieu. Elle ne s'applique pas aux canaux oú il y a un débit moyen d'eau non nul dans une section.

La réciprocité est physiquement assez évidente dans le cas de la houle. Par ailleurs, les équalions de la houle à trois dimensions, même avec un terme de frottement, montrent de suite que le théorème de réciprocité est applicable. On peut par exemple vérifier ce fait de suite en consultant le livre: Methoden der mathematischen Physik, de MM. Courant et Hilbert.

Pour éviter au lecteur de consulter ce livre, nous allons rapidement décrire le raisonnement ici. Pour simplifier les écritures, nous ne considérons que le cas des obstacles sans dissipation d'énergie.

Soit $D$ un domaine contenant l'obstacle, le fluide environnant et deux parties du canal situées de part et d'autre de l'obstacle (fig. 12).

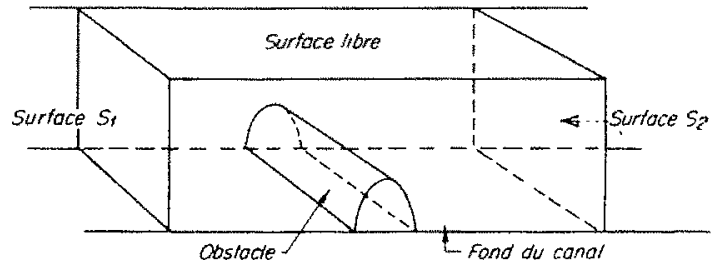

Fia. 12

L'écoulement de l'eau dans $\mathrm{D}$ est régi par :

$$
\Delta_{3} \varphi=0 \text { dans } \mathrm{D}\left(\Delta_{3}=\frac{\partial^{2}}{\partial x^{2}}+\frac{\partial^{2}}{\partial y^{2}}+\frac{\partial^{2}}{\partial z^{2}}\right)
$$

$g \frac{\partial p}{\partial y}+\omega^{2} \varphi=0$ : la surface libre,

$$
\begin{gathered}
\frac{\partial p}{\partial y}=0 \text { an fond el sur les parois du canal } \\
\text { et de lobstacle. }
\end{gathered}
$$

Nous allons appliquer à $D$ la relation de Green-Ostrogradski, $\varphi_{1}$ et $\varphi_{2}$ étant à priori des Conctions quelconques de $x, y, z$.

$\int_{V}\left(\varphi_{1} \Delta_{3} \varphi_{2}-\varphi_{2} \Delta_{3} \varphi_{1}\right) d v=\int_{\mathrm{S}}\left(\varphi_{1} \frac{\partial \varphi_{2}}{\partial n}-\varphi_{2} \frac{\partial \varphi_{1}}{\partial n}\right) d$

V: Volume de D; S : Surface limitant D.

Si $\varphi_{1}$ et $\varphi_{2}$ sont tels que $\Delta \varphi_{1}=\Delta \varphi_{2}=0$ dans $D$, on en tire : :

$$
\int_{s} \varphi_{1} \frac{\partial \varphi_{2}}{\partial n} d \sigma=\int_{s} p_{2} \frac{\partial \varphi_{1}}{\partial n} d \sigma
$$

Si $\varphi_{1}$ et $\varphi_{2}$ satisfont également aux conditions aux limites, les intégrales de surface sont nulles sur le fond, sur les parois du canal et sur l'obstacle puisque $\partial \varphi / \partial n$ y est nul. Si $\varphi_{1}$ et $\varphi_{2}$ satisfont aussi à la condition imposée à la surface libre, on y obtient :

$$
\int_{\mathrm{s}} \varphi_{1} \frac{\partial \varphi_{2}}{\partial n} d \sigma=-\frac{\omega^{2}}{g} \int_{\mathrm{s}} \varphi_{1} \varphi_{2} d \sigma
$$

Donc les deux intégrales sont égales et se détruisent mutuellement.

Il reste donc :

$$
\int_{s_{1}+s_{2}} \varphi_{2} \frac{\partial \rho_{1}}{\partial n} d \sigma=\int_{s_{1}+s_{2}} \rho_{1} \frac{\partial \rho_{2}}{\partial n} d \sigma
$$

$S_{1}$ ef $S_{2}$ : Sections du canal limitant $D$.

Si on fait abstraction des mouvements \& transitoires dans l'espace » produits par l'obstacle, et si on remplace $\varphi_{1}$ et $\varphi_{2}$ par des quantités complexes proportionnelles : $a_{1}$ el $a_{22}$ représentant la hauteur verticale des ondes, et $\left(\partial \varphi_{1} / \partial n\right)$ et $\left(\partial \varphi_{2} / \partial n\right)$ par les quantités complexes $Q_{1}$ et $Q_{2}$ proportionnelles aux débits, on obtient :

$$
\left(a_{1} Q_{2}\right)_{1}+\left(a_{1} Q_{2}\right)_{2}=\left(a_{2} Q_{1}\right)_{1}+\left(a_{2} Q_{1}\right)_{2}
$$

Le premier indice caractérise $\varphi$ et le deuxième la surface $S_{1}$ ou $S_{2}$ considérée.

Admettons que la première fonction $\alpha_{1}$ corresponde à une onde incidente renant de la gauche, done :

$$
\begin{array}{ll}
\left(a_{1}\right)_{1}=1+\beta_{1} & \left(Q_{1}\right)_{1}=1 \cdots \beta_{1} \\
\left(a_{1}\right)_{2}=\alpha_{1} & \left(Q_{1}\right)_{2}=\cdots \cdots \alpha_{1}
\end{array}
$$


Les signes de $\left(Q_{1}\right)_{1}$ et $\left(Q_{1}\right)_{2}$ proviennent de l'orientation de la normale dans : $\partial \varphi / \partial n$.

Si la deuxieme fonction $\phi_{2}$ correspond à une onde incidente venant de la droile, on a pareillement :

$$
\begin{array}{ll}
\left(\alpha_{1}\right)_{1}=\alpha_{2} & \left(Q_{2}\right)_{1}=-\alpha_{2} \\
\left(a_{2}\right)_{2}=1+\beta_{2} & \left(Q_{2}\right)_{2}=1--\beta_{2}
\end{array}
$$

En appliquant la relation (1), on oblient de suite :

$$
\alpha_{1}=\alpha_{2}=\alpha
$$

Remarquons encore une fois que cette relalion est vraie, que l'obstacle soit court ou long, dissipatif ou non.

Dans le cas des obstacles non dissipatifs, on peut, par exemple, considérer un état de mouvement caractérisé par :

$$
\begin{array}{ll}
(a)_{1}=1+\beta_{1}+k \alpha_{2} & (Q)_{1}=1-\beta_{1}-k \alpha_{2} \\
(a)_{2}=\alpha_{1}+k\left(1+\beta_{2}\right) & (Q)_{2}=-\alpha_{1}+k\left(1-\beta_{2}\right)
\end{array}
$$

La conservation de l'énergie se traduit par :

$$
\left.\mathfrak{R}[a)_{1}(\overrightarrow{\mathrm{Q}})_{1}+(a)_{2}(\overrightarrow{\mathrm{Q}})_{2}\right]=0
$$

quel que soit $k$ (réel). $\mathscr{R}[\ldots]$ signifiant partie réelle de ... et $\bar{Q}$ étant la partie conjuguée de $Q$.

On en déduit, $\bar{\alpha}$ et $\bar{\beta}$ étant les parties conjuguées de $\alpha$ et $\beta$ :

$$
\begin{array}{r}
0=\mathcal{R}\left[1-\alpha_{1} \bar{\alpha}_{1}-\beta_{1} \bar{\beta}_{1}+k^{2}\left(1-\alpha_{2} \bar{\alpha}_{2}-\beta_{2} \bar{\beta}_{2}\right)\right. \\
\left.-k\left(\alpha_{2} \vec{\beta}_{1}+\bar{\alpha}_{2} \beta_{1}+\alpha_{1} \bar{\beta}_{2}+\vec{\alpha}_{1} \beta_{2}\right)\right]
\end{array}
$$

D'où :

$$
\begin{gathered}
1-\alpha_{1} \bar{\alpha}_{1}-\beta_{1} \vec{\beta}_{1}=0 \\
1-\alpha_{2} \bar{\alpha}_{2}-\beta_{2} \bar{\beta}_{2}=0 \\
\mathcal{R}\left[\alpha_{2} \bar{\beta}_{1}+\bar{\alpha}_{2} \beta_{1}+\alpha_{1} \bar{\beta}_{2}+\bar{\alpha}_{1} \beta_{2}\right]=0 .
\end{gathered}
$$

Compte lenu de : $\quad \alpha_{1}=\alpha_{2}$

On en déduit :

$$
\begin{gathered}
\beta_{1} \bar{\beta}_{1}=\beta_{2} \vec{\beta}_{2} \\
\mathcal{\alpha}\left[\alpha\left(\overline{\beta_{1}+\beta_{2}}\right)+\bar{\alpha}\left(\beta_{1}+\beta_{2}\right)\right]=0 \\
\alpha\left[\alpha\left(\overline{\beta_{1}+\beta_{2}}\right)\right]=0
\end{gathered}
$$

La deuxième condition montre que $\alpha$ est perpendiculaire à la bissectrice de $\beta_{1}$ et $\beta_{2}$.

Jusqu'à présent, on a gardé les deux plans de référence $S_{1}$ et $S_{2}$. Comme dans l'article de MM. Biesel et Le Méhauté on peut changer de plan de référence, on peut d'abord les réunir puis choisir le plan unique pour confondre $\beta_{1}$ et $\beta_{2}$ puisque dans ce cas $\beta_{1} \bar{\beta}_{1}=\beta_{2} \tilde{\beta}_{2}$. Dans ce cas, on a donc $\beta_{1}=\beta_{2}=\beta$, avec $\beta$ perpendiculaire à $\alpha$.

Dans le cas des obstacles courts (dissipatifs ou non) on peut facilement montrer que $\beta_{1}=\beta_{2}$ si on choisit convenablement les plans de référence. En effet, on a par définition :

$$
\left(Q_{1}\right)_{1}+\left(Q_{1}\right)_{2}=0
$$

l'où :

$$
1-\alpha_{1}-\beta_{1}=0
$$

et de même :

$$
1-\alpha_{2}-\beta_{2}=0
$$

Ceci donne avec $\alpha_{1}=x_{23}: \beta_{1}=\beta_{2}$.

C'est seulement pour les obstacles à la fois relativement longs par rapport à la longueur d'onde et dissipatifs que l'on peul avoir $\left|\beta_{1}\right| \neq\left|\beta_{2}\right|$ et que l'on ne peut pas faire coincider les vecteurs $\beta_{1}$ et $\beta_{2}$, quels que soient les plans de référence choisis. 